\title{
Age-related differences in serum MFG-E8, TGF- $\beta 1$ and correlation to the severity of atherosclerosis determined by ultrasound
}

\author{
HUIYING ZHAO ${ }^{1}$, HUANHUAN ZHANG ${ }^{2}$ and XIUJIAO QIN ${ }^{1}$ \\ ${ }^{1}$ Department of Geriatrics, The First Hospital of Jilin University, Changchun, Jilin 130000; ${ }^{2}$ Department of \\ Intensive Cardiac Care Unit, The Seventh People's Hospital of Zhengzhou, Henan 450006, P.R. China
}

Received May 9, 2017; Accepted October 3, 2017

DOI: $10.3892 / \mathrm{mmr} .2017 .7838$

\begin{abstract}
Atherosclerosis (AS) is an age-related inflammatory disease. Globule-epidermal growth factor-8 (MFG-E8) and transforming growth factor- $\beta 1$ (TGF- $\beta 1$ ) are involved in the pathogenesis of AS. However, age-related changes in circulating levels of MFG-E8 and TGF- $\beta 1$, and their correlation with the severity of AS is not well-characterized. In this study, we investigated age-related changes in serum levels of MFG-E8, TGF- $\beta 1$ and examined their association with the severity of AS. Sixty healthy volunteers were divided into young, middle-age and old-age groups. In addition, carotid ultrasound examination was performed to assess the intima-media thickness (IMT) of carotid artery. Sixty-seven patients with carotid AS and 30 age-matched healthy persons were divided into IMT increased group, plaque group and control group. Serum levels of MFG-E8, TGF- $\beta 1$, tumor necrosis factor- $\alpha$ (TNF- $\alpha)$ and intercellular adhesion molecule-1 were measured in all subjects. A positive association between serum MFG-E8 levels and age was observed in healthy volunteers, while a significant negative association was observed between TGF- $\beta 1$ levels and age. Serum levels of MFG-E8 and TNF- $\alpha$ showed a positive correlation while those of TGF- $\beta 1$ showed a negative correlation with Crouse scores for carotid artery IMT $(\mathrm{P}<0.05$ for both). Both MFG-E8 and TGF- $\beta 1$ were age-related inflammatory factors. MFG-E8 showed a positive correlation, while TGF- $\beta 1$ showed a negative correlation with the severity of AS. Our findings suggest that both MFG-E8 and TGF- $\beta 1$ are age-related inflammatory factors and are related to the degree of AS. In conclusion, both MFG-E8 and TGF- $\beta 1$ may serve as potential markers of the severity of AS.
\end{abstract}

Correspondence to: Dr Huiying Zhao, Department of Geriatrics, The First Hospital of Jilin University, 71 Xinmin Road, Changchun, Jilin 130000, P.R. China

E-mail: zhaohuiying163@163.com

Key words: globule-epidermal growth factor-8, transforming growth factor- $\beta 1$, tumor necrosis factor- $\alpha$, intercellular cell adhesion molecule-1, carotid atherosclerosis

\section{Introduction}

Aging is a process of anatomical, functional and metabolic changes that affects all systems, involves a process of programmed cell death, and modulated by a subtle balance between pro-and antiapoptotic molecules (1). Advancing age is a risk factor for a variety of chronic health problems including cancer, diabetes, cardiovascular, neurodegenerative and musculoskeletal diseases (2). Physical activity and high-Tryptophan diet may play important roles in aging-induced diseases $(3,4)$. Recent studies indicate that declined autophagic capacity in aging cells impairs the process of cellular housekeeping and increases the generation of reactive oxygen species (ROS), which promotes oxidative stress. Oxidative stress can induce the assembly of multiprotein inflammatory complexes called the inflammasomes (5). A growing body of evidence indicates that the aging process is accompanied by a progressive chronic inflammatory response that are mediated via chemokines, adhesion molecules, interleukin-1 (IL-1) and tumor necrosis factor- $\alpha$ (TNF- $\alpha)$. Indeed, the subclinical chronic inflammatory process associated with the aging process has been referred to as 'inflamm-aging' $(6,7)$. Studies have shown that these inflammatory factors are involved in the initiation and regulation of most age-related diseases (8).

Atherosclerosis (AS) is a manifestation of vascular aging and is recognized as a chronic inflammatory disease of the blood vessels. It is one of the underlying pathological changes involved in age-related diseases (9). Inflammatory factors, such as TNF- $\alpha$ and intercellular adhesion molecule-1 (ICAM-1) play important roles in the pathogenesis and progression of AS $(10,11)$.

Age-related oxidative stress mediates chronic inflammation, which further accelerates AS (12). In particular, AS is the common pathophysiological basis of cardiovascular and cerebrovascular diseases. The arterial intima is the first site to be involved in the early stages of AS (13). Intima-media thickness (IMT) is widely used as a surrogate indicator of asymptomatic AS. Increased IMT is an early sign of AS (13). However, plaque formation is the hallmark of AS.

Carotid artery has a greater predilection for development of arteriosclerosis. Atherosclerotic changes in the carotid artery tend to precede those in other parts of the systemic 
vasculature. Studies have shown a close association of carotid AS with coronary artery disease and ischemic cerebrovascular disease. In addition, color Doppler ultrasound examination of carotid artery is a convenient noninvasive and relatively straightforward investigation. Therefore, assessment of carotid intima-media thickness (CIMT) by ultrasonography can be used as a parameter to assess carotid AS. Increased CIMT is an important risk factor for cardiovascular and cerebrovascular diseases and has been used as a noninvasive clinical indicator of early AS (13).

Transforming growth factor- $\beta 1$ (TGF- $\beta 1$ ) is implicated in a wide array of cardiovascular pathological processes, including hypertension, AS, myocardial hypertrophy and heart failure. It is a pleiotropic cytokine which plays multiple roles in the regulation of vascular function and hemostasis (14). In the context of AS, the role of TGF- $\beta 1$ is not well-characterized. On the one hand, TGF- $\beta 1$ is considered to be a protective cytokine (15), which has an antiatherogenic effect, especially in the early stages of AS (16). On the other hand, proatherogenic effects of TGF- $\beta 1$ in the late stages of the disease has also been reported; these include excessive formation of extracellular matrix, promotion of in-stent restenosis and pathological vascular remodeling $(17,18)$.

Globule-epidermal growth factor-8 (MFG-E8) was initially identified as a lactadherin with many discoidin domains secreted in mouse mammary epithelial cells. Recently, studies have shown that MFG-E8 is an element of the arterial inflammatory signaling network which facilitates apoptosis in endothelial cells (19). Studies have shown that MFG-E8 is an inflammatory mediator that orchestrates diverse cellular interactions involved in the pathogenesis of various diseases, including hypertension and AS (19-21).

Recently, high-throughput proteomic screening has indicated high expression levels of MFG-E8 and TGF- $\beta 1$ in aging arterial walls in both mice and humans. Both MFG-E8 and TGF- $\beta 1$ are essential elements of angiotensin II (Ang II) signaling. Upregulation of MFG-E8 and TGF- $\beta 1$ in arterial walls was shown to accelerate age-associated arterial remodeling via activation of Ang II signaling $(19,22)$. In addition, high expression levels of MFG-E8 and TGF- $\beta 1$ have also been found in atherosclerotic plaques (23). However, the age-related differences in circulating MFG-E8, TGF- $\beta 1$ have not been investigated. Whether serum levels of MFG-E8, TGF- $\beta 1$ affect the process of AS is not clear. Further, the association of MFG-E8 and TGF- $\beta 1$ serum levels with severity of AS is not known. We hypothesized that both MFG-E8 and TGF- $\beta 1$ are age-related inflammatory factors and that their expression levels are related to the degree of AS.

In the present study, the primary objective was to examine the association of serum levels of MFG-E8, TGF- $\beta 1$, TNF- $\alpha$ and ICAM-1 with age. The secondary objectives were to determine serum levels of MFG-E8, TGF- $\beta 1$ in patients with AS and to investigate the correlation of serum levels of MFG-E8, TGF- $\beta 1$ with the severity of AS.

\section{Materials and methods}

Ethical approval. The aim of this study and all risks associated with participation in the study were explained to all prospective enrollees and written informed consent was obtained from all subjects prior to their enrolment. The study protocol was approved by the Medical Ethics Committee of the First Hospital of Jilin University (ethical approval no. 2014-306; approval date, August 7,2017) and was in compliance with the principles enshrined in the Declaration of Helsinki.

Study population. A total of 157 study subjects (60 healthy volunteers, 67 patients with carotid AS and 30 age-matched controls without carotid AS) were recruited from the medical examination center at The First Hospital of Jilin University between September and December 2014. All study participants underwent following investigations: Measurement of blood pressure, fasting blood glucose (FBG), blood cell analysis, serum lipid profile [total cholesterol (TC), high-density lipoprotein cholesterol (HDL-C), low-density lipoprotein cholesterol (LDL-C), and triglycerides (TG)], and serum alanine aminotransferase (ALT) levels.

Inclusion criteria. i) Sixty healthy volunteers (29 women and 31 men; age range, 30-74 years) who showed no signs of carotid AS on ultrasound were recruited from the medical examination center at the First Hospital of Jilin University. None of the volunteers had current or past history of hypertension, diabetes mellitus, hyperlipidemia, smoking and other AS risk factors. ii) Sixty seven patients (33 women and 34 men, age range, 40-86 years) with carotid AS diagnosed by ultrasound, but who had normal serum lipid profile, FBG and serum ALT levels.

Exclusion criteria. Patients with carotid AS who qualified any of the following criteria were excluded: i) Use of hormones or other immunosuppressive agents in the immediately preceding one month; ii) abnormal thyroid function, connective tissue disease, Takayasu arteritis or carotid aneurysm; iii) systemic immune disorders, immunosuppressed patients; iv) history of infection of respiratory tract, digestive tract or urinary tract within the last one month; those having symptoms suggestive of infection such as fever, cough, phlegm, nausea, vomiting, diarrhea, abdominal pain, frequent urination, urgency or dysuria; patients with chronic infectious diseases; v) severe anemia, primary hematological diseases; vi) any tumor; vii) chronic hepatitis, liver cirrhosis, sever renal or hepatic insufficiency, acute or chronic pancreatitis; viii) initial stroke, pulmonary infarction or peripheral vascular disease; and ix) organ or bone marrow transplant recipients.

Experimental groups. i) In order to investigate the age-related changes in expressions of MFG-E8 and TGF- $\beta 1,60$ healthy volunteers without carotid AS were divided into 3 groups according to age: Young group $(n=20,10$ men and 10 women; age range, 30-44 years); middle age group $(n=20,10$ men and 10 women; age range, 45-59 years) and old group $(n=20$, 9 men and 11 women; age 60-74 years). ii) To examine a potential association of MFG-E8 and TGF- $\beta 1$ with the degree of AS, 97 patients with carotid AS were divided into 3 groups according to the degree of carotid AS: IMT increased group $(n=27,10$ hypertension, 5 diabetes, 14 without hypertension or diabetes, 2 concomitant hypertension and diabetes); plaque group ( $\mathrm{n}=40,15$ hypertension, 12 diabetes, 
Table I. Baseline characteristics of healthy volunteers.

\begin{tabular}{lccc}
\hline & & Groups & \\
\cline { 2 - 4 } Indicators & Young group $(\mathrm{n}=20)$ & Middle-age group $(\mathrm{n}=20)$ & Old group $(\mathrm{n}=20)$ \\
\hline Age $($ years $)$ & $38.35 \pm 4.15$ & $50.90 \pm 4.01^{\mathrm{b}}$ & $63.35 \pm 3.00^{\mathrm{b}, \mathrm{c}}$ \\
Sex $(\mathrm{male}, \%)$ & $10(50.0 \%)$ & $10(50.0 \%)$ & $9(45.0 \%)$ \\
SBP $(\mathrm{mmHg})$ & $121.9 \pm 9.0$ & $129.4 \pm 11.7$ & $128.7 \pm 12.2$ \\
DBP $(\mathrm{mmHg})$ & $78.7 \pm 5.9$ & $78.1 \pm 8.1$ & $80.2 \pm 8.0$ \\
HR $(\mathrm{beats} / \mathrm{min})$ & $79.1 \pm 12.0$ & $73.4 \pm 12.3$ & $71.9 \pm 9.2$ \\
WBC $\left(\mathrm{x} 10^{9} / \mathrm{l}\right)$ & $6.48 \pm 1.10$ & $6.39 \pm 1.19$ & $6.84 \pm 1.37$ \\
NE $(\%)$ & $0.58 \pm 0.07$ & $0.57 \pm 0.06$ & 0.067 \\
FPG $(\mathrm{mmol} / \mathrm{l})$ & $5.32 \pm 0.34$ & $5.40 \pm 0.50$ & $5.59 \pm 0.08$ \\
TG $(\mathrm{mmol} / \mathrm{l})$ & $1.67 \pm 0.87$ & $1.63 \pm 1.02$ & $1.44 \pm 0.76$ \\
TC $(\mathrm{mmol} / \mathrm{l})$ & $4.56 \pm 0.64$ & $4.70 \pm 0.84$ & 0.111 \\
HDL-C $(\mathrm{mmol} / \mathrm{l})$ & $1.29 \pm 0.35$ & $1.35 \pm 0.34$ & 0.463 \\
LDL-C $(\mathrm{mmol} / \mathrm{l})$ & $2.53 \pm 0.54$ & $2.77 \pm 0.65$ & 0.712 \\
Cr $(\mu \mathrm{mol} / \mathrm{l})(\mu \mathrm{mol} / \mathrm{l})$ & $64.52 \pm 13.69$ & $70.27 \pm 15.92$ & 0.336 \\
Uric acid $(\mu \mathrm{mol} / \mathrm{l})$ & $327.10 \pm 83.86$ & $357.45 \pm 98.97$ & $0.95^{\mathrm{a}}$ \\
\hline
\end{tabular}

SBP, systolic pressure; DBP, diastolic pressure; HR, heart rate; WBC, white blood cell; NE, neutrophils cell; FPG, fasting plasma glucose; TG, total cholesterol; TC, total glyceride; HDL-C, high-density lipoprotein cholesterol; LDL-C, low-density lipoprotein cholesterol; Cr, creatinine. ${ }^{a} \mathrm{P}<0.05$ vs. young group; ${ }^{b} \mathrm{P}<0.001$ vs. young group; ${ }^{\mathrm{c}} \mathrm{P}<0.001 \mathrm{vs}$. middle-age group.

21 without hypertension or diabetes, 8 concomitant hypertension and diabetes) and control group ( $\mathrm{n}=30,12$ hypertension, 6 diabetes, 15 without hypertension or diabetes, 9 concomitant hypertension and diabetes).

Carotid ultrasound acquisition protocol and diagnostic criteria for carotid AS. All enrolled participants underwent carotid ultrasound examination with an ultrasonic probe frequency of $7.0 \mathrm{MHz}$ by a trained senior technician from the color ultrasonic room, Department of Neurology at the First Hospital of Jilin University First Hospital. Carotid artery ultrasound examinations were performed as previously described (24) with some modifications. In brief, for each patient, ultrasonic images were obtained from 10 segments of the bilateral carotid arteries: The common carotid artery, the carotid bifurcation, the internal carotid artery, external carotid artery and subclavian artery. CIMT of bilateral common carotid arteries were measured. The maximal CIMT was measured on the near and far wall at each segment. According to the diagnostic criteria for carotid AS recommended by the Ultrasound Physicians Society of China (2011), IMT $<1.0 \mathrm{~mm}$ was defined as normal; $1.0 \mathrm{~mm}<$ IMT $<1.5 \mathrm{~mm}$ was defined as increased CIMT; local CIMT $\geq 1.5 \mathrm{~mm}$ or a relative $50 \%$ increase of the local intima thickness was defined as carotid atherosclerotic plaque. Therefore, patients with increased CIMT or carotid atherosclerotic plaque or both were diagnosed as CAS.

Evaluation of carotid plaque by Crouse score. Leaving the length of carotid atherosclerotic plaques out of account, Crouse scores for plaques were determined as the sum of the maximum IMT of each plaque of both right and left carotid arteries, as described elsewhere (25).
Enzyme-linked immunosorbent assays for serum MFG-E8, $T G F-\beta 1, T N F-\alpha$ and ICAM-1. Plasma concentrations of MFG-E8, TGF- $\beta 1$, TNF- $\alpha$ and ICAM- 1 were determined by ELISA kits (cat. no. 2805-MF-050, MFG-E8, Solid Phase Sandwich ELISA; R\&D Systems, Minneapolis, MN, USA), (cat. no. SEA010Hu, TGF- $\beta 1$ ), (cat. no. SEA133Hu, TNF- $\alpha$ ), (cat. no. SEA548Hu, ICAM-1) (all from Uscn Life Science, Inc., Wuhan, China), following the manufacturer's instructions. The minimal detectable concentrations were: $0.5 \mathrm{pg} / \mathrm{ml}$ for MFG-E8; $1.0 \mathrm{pg} / \mathrm{ml}$ for TGF- $\beta 1 ;<4.0 \mathrm{pg} / \mathrm{ml}$ for TNF- $\alpha$; and $1.0 \mathrm{pg} / \mathrm{ml}$ for ICAM-1.

Laboratory measurements. TC, TGs, HDL-C, and FBG were quantified by routine enzymatic laboratory methods using an automated biochemical analyzer (Hitachi 7060; Hitachi, Tokyo, Japan).

Statistical analysis. A database was built using SPSS 20.0 for statistical analysis. Normally distributed continuous variables are presented as mean $\pm \mathrm{SD}$, while non-normally distributed variables are presented as median and 25-75th interquartile range. Categorical variables are presented as frequencies or percentages. For quantitative continuous variables, the independent samples t-test was performed. One-way ANOVA was used to assess the differences between the three groups. Q test was used to assess between-group differences. Chi-square test was used to compare categorical variables. Pearson correlation test was used for normally distributed continuous variables, while Spearman correlation test was used for non-normally distributed continuous variables. Logistic regression analysis was performed to describe the relationship of MFG-E8 and TGF- $\beta 1$ with the degree of $\mathrm{AS} . \mathrm{P}<0.05$ was considered to indicate a statistically significant difference. 
Table II. Serum levels of MFG-E8, TGF- $\beta 1$, TNF- $\alpha$ and ICAM-1 in healthy volunteers disaggregated into three age-groups.

\begin{tabular}{lccc}
\hline & \multicolumn{3}{c}{ Groups } \\
\cline { 2 - 4 } Indicators & Young group $(\mathrm{n}=20)$ & Middle-age group $(\mathrm{n}=20)$ & Old group $(\mathrm{n}=20)$ \\
\hline MFG-E8 $(\mathrm{pg} / \mathrm{ml})$ & $4080.5298 \pm 1090.88$ & $4355.45 \pm 1037.28$ & $5367.32 \pm 2297.79^{\mathrm{a}}$ \\
TGF- $\beta 1(\mathrm{pg} / \mathrm{ml})$ & $3201.33 \pm 576.05$ & $2672.20 \pm 959.81$ & $2219.81 \pm 918.19^{\mathrm{b}}$ \\
TNF- $\alpha(\mathrm{pg} / \mathrm{ml})$ & $7.19 \pm 3.48$ & $9.25 \pm 4.92$ & $13.28 \pm 6.58^{\mathrm{b}}$ \\
ICAM-1 $(\mathrm{ng} / \mathrm{ml})$ & $321.95 \pm 138.90$ & $428.38 \pm 155.63$ & $534.18 \pm 210.89^{\mathrm{b}}$ \\
\hline
\end{tabular}

${ }^{\mathrm{a} P}<0.01$ vs. young group; ${ }^{\mathrm{b}} \mathrm{P}<0.05$ vs. young group. MFG-E8, globule-epidermal growth factor- 8 ; TGF- $\beta 1$, transforming growth factor- $\beta 1$; TNF- $\alpha$, tumor necrosis factor- $\alpha$; ICAM-1 intercellular adhesion molecule 1 .
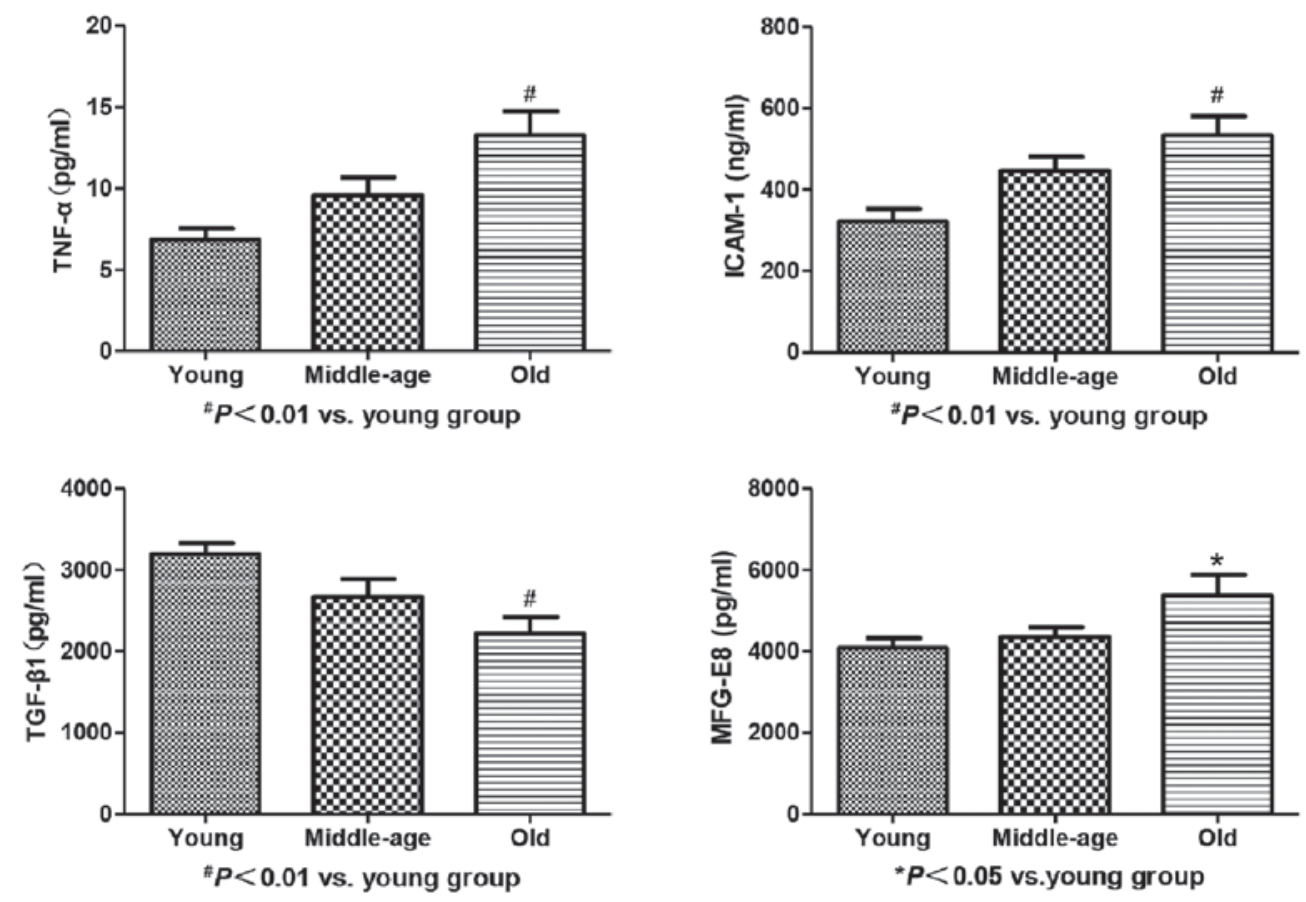

Figure 1. Serum levels of MFG-E8, TGF- $\beta 1$, TNF- $\alpha$ and ICAM-1 in 3 groups of healthy volunteers. ${ }^{*} \mathrm{P}<0.01$ vs. young group, "P<0.05 vs. young group. MFG-E8, globule-epidermal growth factor- 8 ; TGF- $\beta 1$, transforming growth factor- $\beta 1$; TNF- $\alpha$, tumor necrosis factor- $\alpha$; ICAM-1 intercellular adhesion molecule 1.

\section{Results}

Baseline characteristics of healthy volunteers. There was a significant difference in ages of healthy volunteers in the young, middle-age and old groups $(\mathrm{P}<0.001$ for all). Significant differences were found with respect to TC and LDL-C levels between the young, middle-age and old groups $(\mathrm{P}<0.05$ for all). This suggested that TC, and LDL-C levels increased with increase in age. There was no significant difference with respect to other variables between the three groups $(\mathrm{P}>0.05$ for all; Table I).

Serum levels of MFG-E8, TGF- $\beta 1, T N F-\alpha$ and ICAM-1 in healthy volunteers. Serum levels of MFG-E8, TNF- $\alpha$ and ICAM-1 increased gradually with increase in age; however, statistically significant differences in this respect were found only between the young and old groups $\left(\mathrm{P}_{1}<0.05, \mathrm{P}_{2}<0.01\right.$, $\mathrm{P}_{3}<0.01$, respectively). In contrast, there was an increasing trend in TGF- $\beta 1$ level with increase in age; however, a significant difference in this respect was observed only between the young and old groups $(\mathrm{P}<0.01$; Table II and Fig. 1).

Correlation of peripheral MFG-E8,TGF- $\beta 1, T N F-\alpha$ and $I C A M-1$ with age. Spearman correlation analysis revealed a significant positive correlation of serum levels of MFG-E8, TNF- $\alpha$ and ICAM- 1 with age $(\mathrm{r} 1=0.267, \mathrm{p} 1=0.039 ; \mathrm{r} 2=0.483$, $\mathrm{p} 2=0.000 ; \mathrm{r} 3=0.370, \mathrm{p} 3=0.004$, respectively). However, a negative correlation was observed between serum levels of TGF- $\beta 1$ and age $(\mathrm{r}=-0.318, \mathrm{p}=0.013)$. Besides, a negative relationship was found between serum TNF- $\alpha$ and ICAM-1 (r=0.259, p=0.04; Fig. 2).

Baseline characteristics of patients with carotid AS. Serum HDL-C level in plaque group of CAS patients was significantly lower than that in the increased IMT group $(\mathrm{P}<0.01)$. However, there were no significant differences between the three groups with respect to age, sex, history of hypertension, diabetes and smoking ( $\mathrm{P}>0.05$ for all; Table III). 

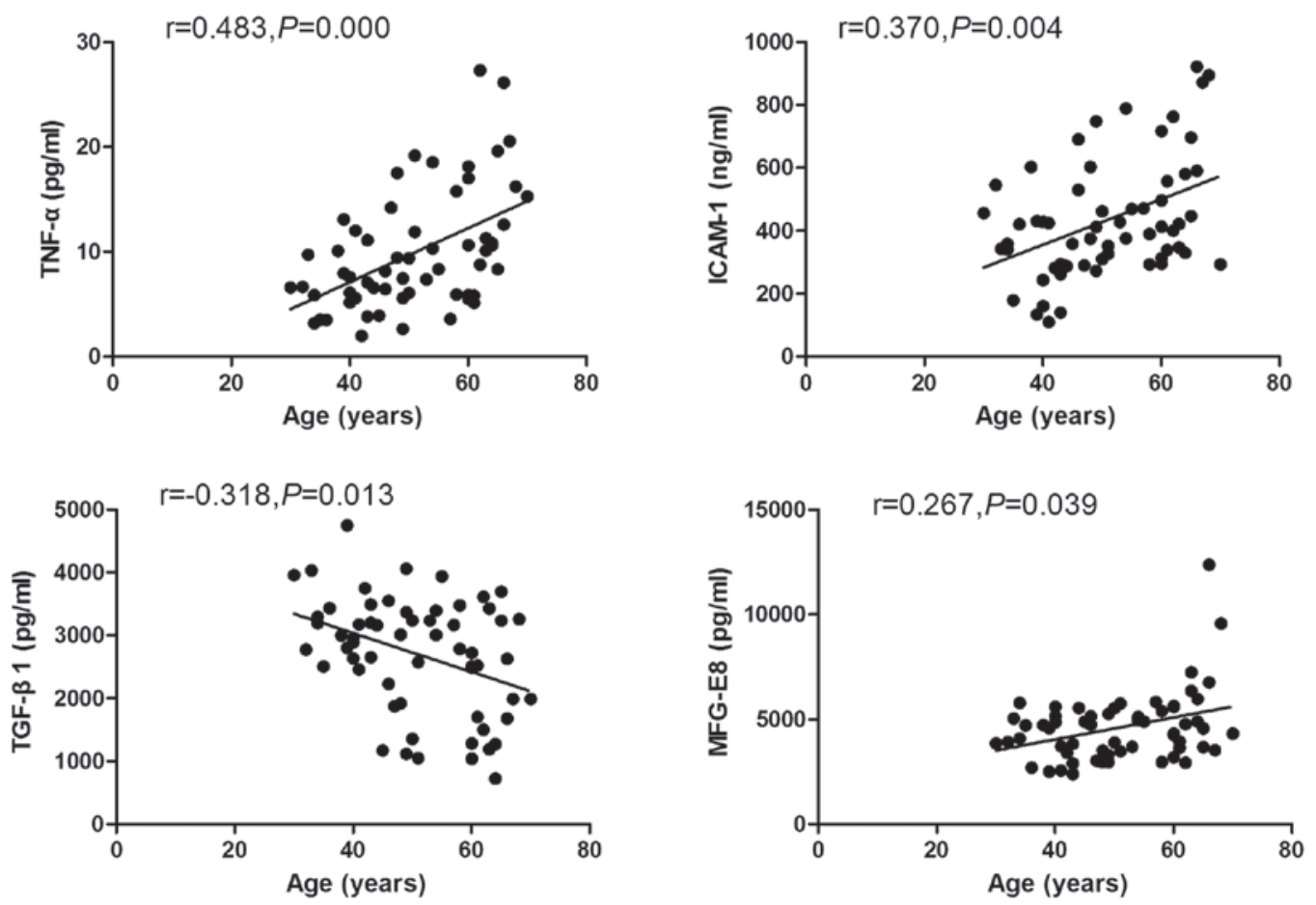

Figure 2. Results of Spearman's correlation analysis showing correlation of serum levels of MFG-E8, TGF- $\beta 1$, TNF- $\alpha$ and ICAM- 1 with age. MFG-E8, globule-epidermal growth factor- 8 ; TGF- $\beta 1$, transforming growth factor- $\beta 1$; TNF- $\alpha$, tumor necrosis factor- $\alpha$; ICAM-1 intercellular adhesion molecule 1 .

Table III. Baseline characteristics of patients with carotid atherosclerosis and controls.

\begin{tabular}{lcccr}
\hline & \multicolumn{3}{c}{ Groups } \\
\cline { 2 - 4 } Indicators & Control $(\mathrm{n}=30)$ & Increased IMT $(\mathrm{n}=27)$ & Plaques $(\mathrm{n}=40)$ & P-value \\
\hline Age (years) & $61.36 \pm 9.01$ & $61.44 \pm 8.14$ & $64.78 \pm 7.78$ & 0.148 \\
Sex (male, $\%)$ & $16(53.3 \%)$ & $9(33.3 \%)$ & $25(62.5 \%)$ & 0.062 \\
Hypertension $(\%)$ & $12(40.0 \%)$ & $10(37.0 \%)$ & $15(37.5 \%)$ & 0.968 \\
Diabetes $(\%)$ & $6(20.0 \%)$ & $5(18.5 \%)$ & $12(30 \%)$ & 0.471 \\
Smoking $(\%)$ & $6(20.0 \%)$ & $4(14.8 \%)$ & $13(32.5 \%)$ & 0.210 \\
SBP $(\mathrm{mmHg})$ & $133.1 \pm 14.3$ & $133.8 \pm 18.4$ & $137.7 \pm 20.5$ & 0.512 \\
DBP $(\mathrm{mmHg})$ & $80.0(74.8,86.3)$ & $80.0(72.0,90.0)$ & $80.0(70.0,84.8)$ & 0.705 \\
HR $(\mathrm{beat} / \mathrm{min})$ & $70.0(67.5,80.0)$ & $71.0(66.0,77.0)$ & $70.0(63.3,75.8)$ & 0.378 \\
WBC $\left(\mathrm{x} 10^{\%} / \mathrm{l}\right)$ & $6.35 \pm 1.45$ & $6.63 \pm 1.40$ & $6.65 \pm 1.61$ & 0.689 \\
NE $(\%)$ & $0.58 \pm 0.06$ & $0.60 \pm 0.07$ & $0.60 \pm 0.08$ & 0.312 \\
FPG $(\mathrm{mmol} / \mathrm{l})$ & $5.47(5.01,6.05)$ & $5.26(5.03,6.21)$ & $5.58(5.19,6.86)$ & 0.326 \\
TG $(\mathrm{mmol} / \mathrm{l})$ & $1.40(1.05,2.31)$ & $1.12(0.85,1.86)$ & $1.28(0.78,2.05)$ & 0.477 \\
TC $(\mathrm{mmol} / \mathrm{l})$ & $4.62 \pm 0.98$ & $4.68 \pm 0.93$ & $4.47 \pm 1.12$ & 0.695 \\
HDL-C $(\mathrm{mmol} / \mathrm{l})$ & $1.27 \pm 0.32$ & $1.40 \pm 0.42$ & $1.15 \pm 0.33^{\mathrm{a}}$ & 0.021 \\
LDL-C $(\mathrm{mmol} / \mathrm{l})$ & $2.72 \pm 0.74$ & $2.63 \pm 0.80$ & $2.61 \pm 0.80$ & 0.826 \\
Cr $(\mu \mathrm{mol} / \mathrm{l})$ & $63.63 \pm 20.17$ & $61.76 \pm 12.38$ & $68.20 \pm 12.44$ & 0.208 \\
Uric acid $(\mu \mathrm{mol} / \mathrm{l})$ & $373.29 \pm 121.15$ & $325.35 \pm 97.30$ & $326.34 \pm 89.49$ & 0.205 \\
\hline
\end{tabular}

SBP, systolic pressure; DBP, diastolic pressure; HR, heart rate; WBC, white blood cell; NE, neutrophils cell; FPG, fasting plasma glucose; TG, total cholesterol; TC, total glyceride; HDL-C, high-density lipoprotein cholesterol; LDL-C, low-density lipoprotein cholesterol; Cr, creatinine. HR, heart rate. ${ }^{\mathrm{a}} \mathrm{P}<0.01$ vs. increased IMT group.

Serum levels of MFG-E8,TGF- $\beta 1, T N F-\alpha$ and ICAM-1 in patients with carotid AS. Serum levels of MFG-E8 in the plaques group were significantly higher than those in the control and increased IMT groups ( $\mathrm{P}<0.05$ for both). This finding suggested a significant increase in MFG-E8 with increase in the degree of AS. On the contrary, serum levels 

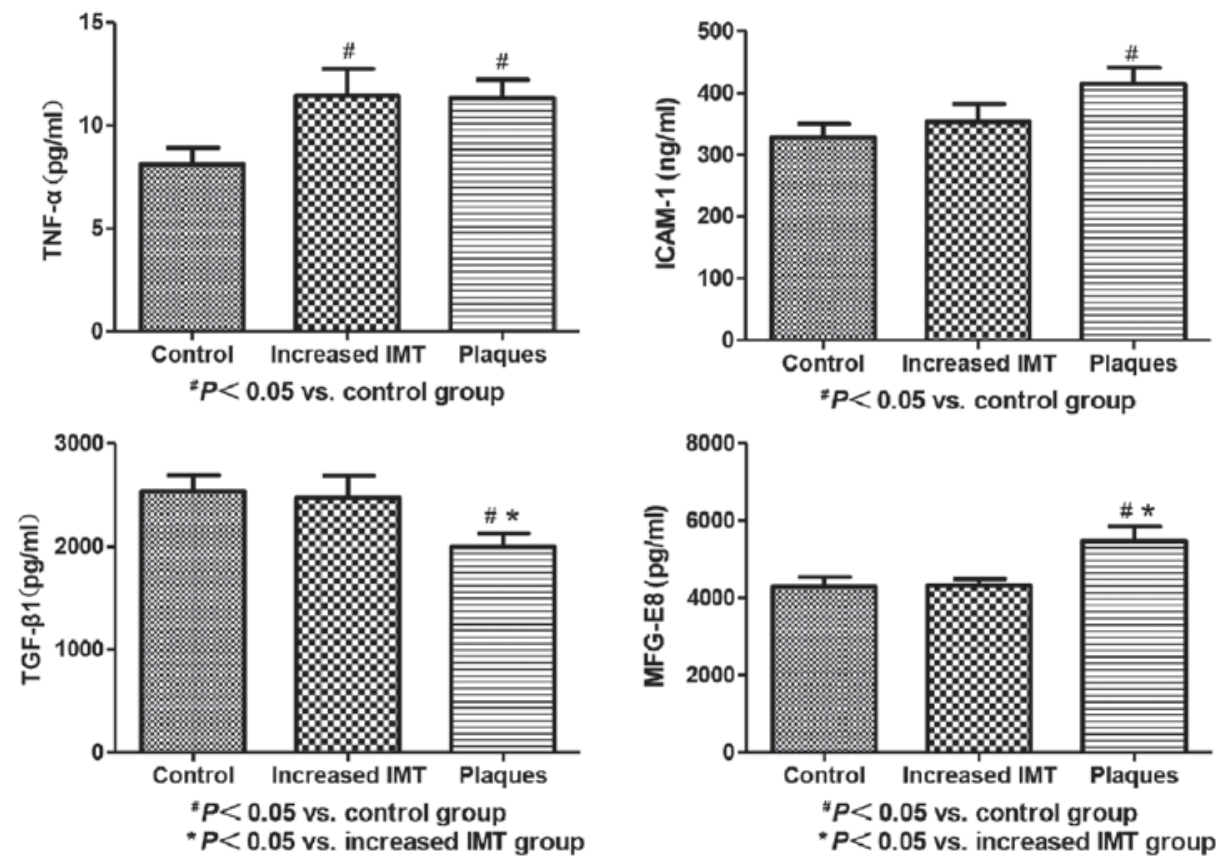

Figure 3. Serum levels of MFG-E8, TGF- $\beta 1$, TNF- $\alpha$ and ICAM-1 in patients with carotid atherosclerosis. ${ }^{.} \mathrm{P}<0.05$ vs. control group, ${ }^{*} \mathrm{P}<0.05$ vs. increased IMT group. MFG-E8, globule-epidermal growth factor- 8 ; TGF- $\beta 1$, transforming growth factor- $\beta 1$; TNF- $\alpha$, tumor necrosis factor- $\alpha$; ICAM-1 intercellular adhesion molecule 1.
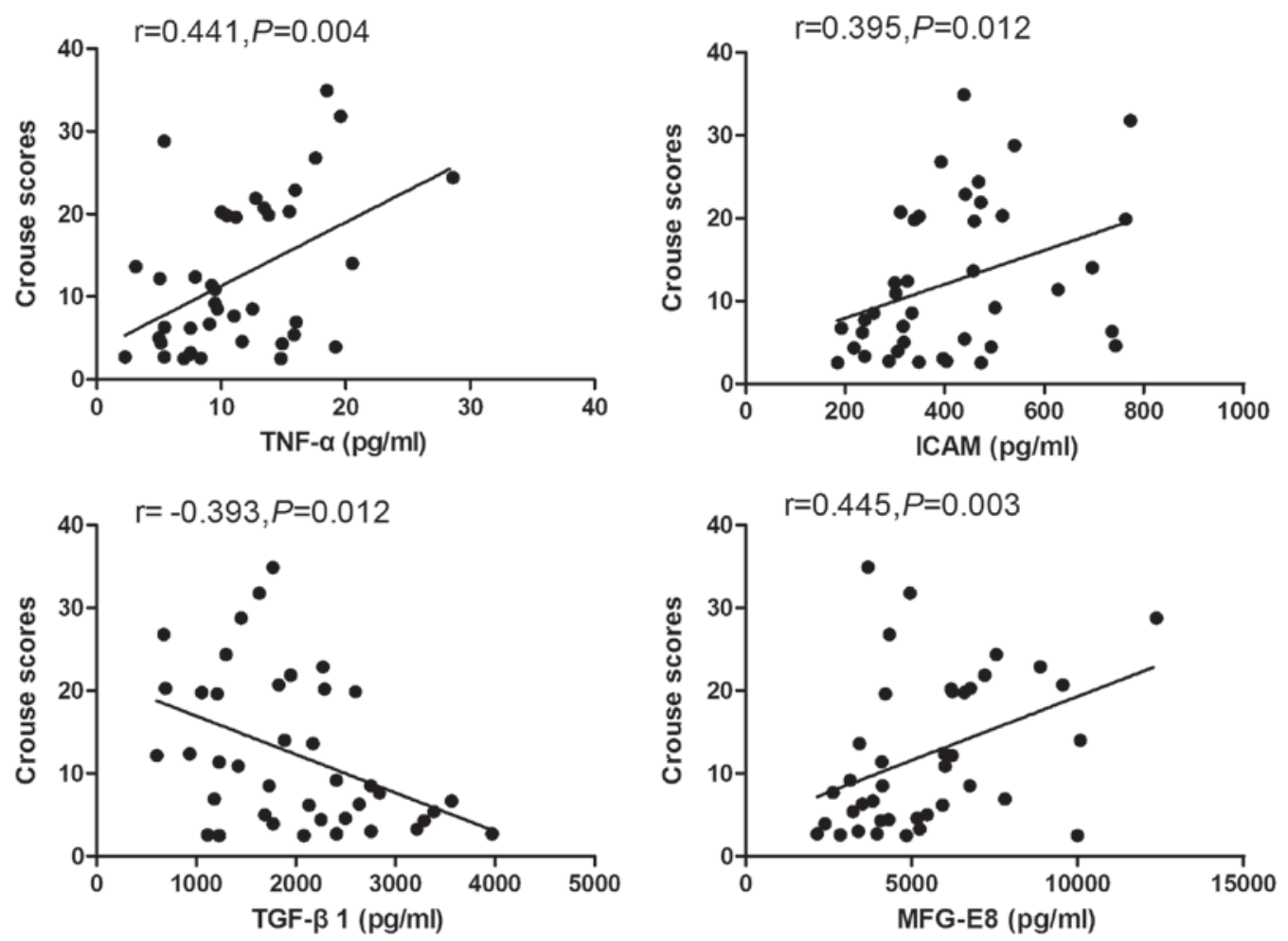

Figure 4. Correlations of Crouse scores with serum levels of MFG-E8, TGF- $\beta 1$, TNF- $\alpha$ and ICAM-1 of patients with carotid atherosclerosis. MFG-E8, globule-epidermal growth factor-8; TGF- $\beta 1$, transforming growth factor- $\beta 1$; TNF- $\alpha$, tumor necrosis factor- $\alpha$; ICAM-1 intercellular adhesion molecule 1 .

of TGF- $\beta 1$ in the plaques group were significantly lower than those in the control and increased IMT groups $(\mathrm{P}<0.05$ for both), which suggested that TGF- $\beta 1$ level decreased significantly with increase in the degree of AS. In addition, serum levels of TNF- $\alpha$ in CAD patients, both in increased IMT and plaques groups, were significantly higher than that in the control group ( $\mathrm{P}<0.05$ for both); however, there was no significant difference between the IMT and plaques groups in this respect $(\mathrm{P}>0.05)$. Serum levels of ICAM-1 in the plaques group were higher than those in the control group $(\mathrm{P}<0.05)$; however, there was no significant difference in this respect between the IMT and plaques groups ( $\mathrm{P}>0.05$; Fig. 3 ). 
Correlation of Crouse scores with serum levels of $M F G-E 8$, $T G F-\beta 1, T N F-\alpha$ and ICAM-1. Crouse scores for carotid artery intima-media thickness were found to be associated with serum levels of MFG-E8, TGF- $\beta 1$ and other inflammatory factors on Spearman analysis. Serum levels of MFG-E8, TNF- $\alpha$ and ICAM-1 showed a significant positive correlation with Crouse scores for carotid artery intima-media thicknesses $(\mathrm{r} 1=0.455$, $\mathrm{r} 2=0.441$ and $\mathrm{r} 3=0.395$, respectively; $\mathrm{P}<0.05$ for all). In contrast, a negative correlation was observed between serum levels of TGF- $\beta 1$ and Crouse scores ( $r=-0.393$, P<0.05; Fig. 4).

\section{Discussion}

AS is an age-related disease and several inflammatory factors participate in the process of AS. Overall, our findings indicate a direct association between serum levels of MFG-E8, TNF- $\alpha$ and ICAM-1 with increase in age. Moreover, serum levels of MFG-E8, TNF- $\alpha$ and ICAM-1 showed a positive correlation, while those of TGF- $\beta 1$ showed a negative correlation with age. In addition, MFG-E8 serum levels in CAD patients were significantly higher in the increased IMT and plaque groups. MFG-E8 levels in plaque group were significantly higher than those in the increased IMT group. On the contrary, serum levels of TGF- $\beta 1$ in CAD patients in the plaque group were lower than those in the control and increased IMT groups. Serum TGF- $\beta 1$ levels in the increased IMT group were significantly lower than those in the control group. On logistic regression analysis, serum levels of MFG-E8, TNF- $\alpha$ and ICAM-1 showed a significant positive relation with Crouse score for carotid artery intima-media thickness. In contrast, serum levels of TGF- $\beta 1$ showed a significant negative relation with Crouse score.

Few studies have reported the correlation between serum MFG-E8 and age. Cheng et al described a gradual increase in MFG-E8 with age and a positive correlation between serum MFG-E8 and pulse wave velocity in elderly patients with type 2 diabetes mellitus (26). The findings are consistent with our results on the association between MFG-E8 and increase in age. In this study, we observed a positive correlation between serum levels of MFG-E8 and Crouse score for carotid artery intima-media thickness in patients with carotid AS. This suggested a positive correlation between serum levels of MFG-E8 and the severity of carotid AS as assessed by ultrasound. This finding provides a novel therapeutic target for AS. Based on the previous studies, the likely mechanisms involved in the age-related changes in serum MFG-E8 may be as follows: age-associated activation of Ang II signaling. Upregulation of Ang II signaling within the central arterial wall with increase in age is well-documented (19). This may contribute to upregulation of Ang II, angiotensin-converting enzyme (ACE), Ang II receptor and AT1 receptor, which may induce vascular smooth cells to secrete MFG-E8 (27,28). A second mechanism may be the increased secretion of MFG-E8 by activated macrophages. Studies have shown secretion of MFG-E8 by activated macrophages in response to inflammatory reaction $(29,30)$. Subclinical chronic inflammation with age would increase the pool of activated macrophages, which may lead to increased production of MFG-E8.

In addition, increased MFG-E8 secretion could stimulate MCP-1 expression, which accelerates the release of other inflammatory factors such as TNF- $\alpha$ and ICAM-1 (19). All of these mechanisms may be involved in the process of 'inflammation-aging'.

TGF- $\beta 1$ is secreted by various cells, and is activated by plasmin cleavage. A previous study demonstrated that expression levels of TGF- $\beta 1$ in arterial wall were associated with vascular AS and age. Redondo et al observed an age-associated decrease in expression of TGF- $\beta 1$ in serum and vascular smooth muscle cells (VSMC) isolated from 169 patients who underwent coronary artery bypass grafting (CABG) (31). The degree of cellular senescence was closely related to lower TGF- $\beta 1$ secretion and downregulation of signaling pathway. Contrarily, Kanzaki et al reported increase in TGF- $\beta 1$ level with age. They reported over-expression of TGF- $\beta 1$ in atherosclerotic lesions, and exogenous TGF- $\beta 1$ promoted neointima formation in rabbit (32). Furthermore, TGF- $\beta 1$ was shown to enhance foam cell formation by inducing downregulation of CD36 and scavenger receptor A in macrophages of atherosclerotic plaques via a reparative response to vascular injury (33). The findings pertaining to TGF- $\beta 1$ in the present study are similar to those reported previously (31). It may contribute to vascular cells senescence. Age-induced senescence of vascular cells resulted in decreased endogenous secretion of TGF- $\beta 1$, although compensatory effects also play a role in this process. Further research is required to elucidate the underlying mechanisms. This study is the first to demonstrate a negative correlation between TGF- $\beta 1$ and the degree of CAS.

Our study showed a gradual increase in TNF- $\alpha$ and ICAM-1 levels with increase in age; further, their expression levels in the CAS group were higher than those in healthy control, while no significant difference was observed in this respect between the IMT and plaques groups. The findings suggest that both TNF- $\alpha$ and ICAM-1 are age-related inflammatory factors and are associated with AS. Expression levels of both showed a relation with the degree of AS. In a previous study, TNF- $\alpha$ was shown to stimulate ICAM-1 gene transcription and protein expression via activation of the nuclear factor- $\kappa \mathrm{B}$ $(\mathrm{NF}-\kappa \mathrm{B})$ (34). Our study revealed a positive relation between serum levels of TNF- $\alpha$ and ICAM-1 in enrolled healthy volunteers, which is consistent with previous studies. Therefore, these results suggest that TNF- $\alpha$ and ICAM-1 are involved in the age-induced chronic inflammation and interact with each other in the aging process. Moreover, we also observed a positive correlation between serum levels of TNF- $\alpha$ and ICAM-1 and Crouse scores for carotid artery intima-media thickness in CAS group, which is also consistent with the results of a previous study (35). Serum levels of TNF- $\alpha$ and ICAM-1 increased with the increase in the degree of AS, which is consistent with previous researches (36). Based on these results, serum levels of TNF- $\alpha$ and ICAM-1 may also be used to reflect the degree of CAS.

In regards to the limitations of the present study, our research verified the age-related changes in serum levels of MFG-E8 and TGF- $\beta 1$ and their correlation with the severity of CAS; however, there were some study limitations. Firstly, the study population comprised of residents of northeast China. Secondly, the effect of blood lipid levels and drugs on the serum levels of MFG-E8, TGF- $\beta 1$ and other inflammatory factors were not considered. Thirdly, the mechanisms by which MFG-E8 and TGF- $\beta 1$ regulate AS were not investigated. A 
further study will be needed to unravel their mutual interactions and to explore the mechanisms by which these contribute to the pathogenesis and development of AS.

In conclusion, the study demonstrated that both MFG-E8 and TGF- $\beta 1$ were age-related inflammatory factors through examining the association of the serum levels of MFG-E8, TGF- $\beta 1$, TNF- $\alpha$ and ICAM- 1 with age. Further, this is the first study to show a positive correlation between serum MFG-E8 level and the degree of AS as determined by ultrasound. In contrast, TGF- $\beta 1$ level showed a negative correlation with the degree of AS. Our findings are of much clinical relevance and suggest that both MFG-E8 and TGF- $\beta 1$ may serve as quantitative indices of the severity of AS.

\section{Acknowledgements}

This study was supported in part by Natural Foundation of China (project grant no. 51372096) and Natural Foundation of Jilin China (project grant no. 201015143).

\section{References}

1. Green DR and Llambi F: Cell death signaling. Cold Spring Harb Perspect Biol 7: a006080, 2015.

2. Mobasheri A, Matta C, Zákány R and Musumeci G: Chondrosenescence: Definition, hallmarks and potential role in the pathogenesis of osteoarthritis. Maturitas 80: 237-244, 2015.

3. Musumeci G, Castrogiovanni P, Szychlinska MA, Imbesi R, Loreto C, Castorina S and Giunta S: Protective effects of high Tryptophan diet on aging-induced passive avoidance impairment and hippocampal apoptosis. Brain Res Bull 128: 76-82, 2017.

4. Musumeci G, Castrogiovanni P, Trovato FM, Imbesi R, Giunta S, Szychlinska MA, Loreto C, Castorina S and Mobasheri A: Physical activity ameliorates cartilage degeneration in a rat model of aging: A study on lubricin expression. Scand J Med Sci Sports 25: e222-e230, 2015.

5. Salminen A, Ojala J, Kaarniranta K and Kauppinen A: Mitochondrial dysfunction and oxidative stress activate inflammasomes: Impact on the aging process and age-related diseases. Cell Mol Life Sci 69: 2999-3013, 2012.

6. Franceschi C, Bonafè M, Valensin S, Olivieri F, De Luca M, Ottaviani E and De Benedictis G: Inflamm-aging. An evolutionary perspective on immunosenescence. Ann NY Acad Sci 908: 244-254, 2000.

7. Xia S, Zhang X, Zheng S, Khanabdali R, Kalionis B, Wu J, Wan W and Tai X: An update on inflamm-aging: Mechanisms, prevention, and treatment. J Immunol Res 2016: 8426874, 2016.

8. De Martinis M, Franceschi C, Monti D and Ginaldi L: Inflammation markers predicting frailty and mortality in the elderly. Exp Mol Pathol 80: 219-227, 2006.

9. Pelisek J, Wendorff H, Wendorff C, Kuehnl A and Eckstein HH: Age-associated changes in human carotid atherosclerotic plaques. Ann Med 48: 541-551, 2016.

10. Papa A, Danese S, Urgesi R, Grillo A, Guglielmo S, Roberto I, Semeraro S, Scaldaferri F, Pola R, Flex A, et al: Intercellular adhesion molecule 1 gene polymorphisms in inflammatory bowel disease. Eur Rev Med Pharmacol Sci 8: 187-191, 2004

11. Mohammadpour AH, Falsoleiman H, Shamsara J, Allah Abadi G, Rasooli R and Ramezani M: Pentoxifylline decreases serum level of adhesion molecules in atherosclerosis patients. Iran Biomed J 18: 23-27, 2014.

12. Paneni F, Costantino S and Cosentino F: Molecular pathways of arterial aging. Clin Sci (Lond) 128: 69-79, 2015.

13. Gujral DM, Shah BN, Chahal NS, Bhattacharyya S, Hooper J, Senior R, Harrington KJ and Nutting CM: Carotid intima-medial thickness as a marker of radiation-induced carotid atherosclerosis. Radiother Oncol 118: 323-329, 2016.

14. Schuliga M: The inflammatory actions of coagulant and fibrinolytic proteases in disease. Mediators Inflamm 2015: 437695, 2015.

15. Pasterkamp G and Goumans MJ: The microvasculature: The next battlefield where transforming growth factor- $\beta$ and endoglin draw their double-edged swords? Arterioscler Thromb Vasc Biol 37: 10-12, 2017.
16. Dhaouadi N, Li JY, Feugier P, Gustin MP, Dab H, Kacem K, Bricca $\mathrm{G}$ and Cerutti C: Computational identification of potential transcriptional regulators of TGF- $\beta 1$ in human atherosclerotic arteries. Genomics 103: 357-370, 2014.

17. Redondo S, Navarro-Dorado J, Ramajo M, Medina Ú and Tejerina T: The complex regulation of TGF- $\beta$ in cardiovascular disease. Vasc Health Risk Manag 8: 533-539, 2012.

18. Toma I and McCaffrey TA: Transforming growth factor- $\beta$ and atherosclerosis: Interwoven atherogenic and atheroprotective aspects. Cell Tissue Res 347: 155-175, 2012.

19. Wang M, Wang HH and Lakatta EG: Milk fat globule epidermal growth factor VIII signaling in arterial wall remodeling. Curr Vasc Pharmacol 11: 768-776, 2013.

20. Wang M, Fu Z, Wu J, Zhang J, Jiang L, Khazan B, Telljohann R, Zhao M, Krug AW, Pikilidou M, et al: MFG-E8 activates proliferation of vascular smooth muscle cells via integrin signaling. Aging Cell 11: 500-508, 2012.

21. Yi YS: Functional role of milk fat globule-epidermal growth factor viii in macrophage-mediated inflammatory responses and inflammatory/autoimmune diseases. Mediators Inflamm 2016: 5628486, 2016.

22. Wang M, Khazan B and Lakatta EG: Central arterial aging and angiotensin II signaling. Curr Hypertens Rev 6: 266-281, 2010.

23. Bagnato C, Thumar J, Mayya V, Hwang SI, Zebroski H, Claffey KP, Haudenschild C, Eng JK, Lundgren DH and Han DK: Proteomics analysis of human coronary atherosclerotic plaque: A feasibility study of direct tissue proteomics by liquid chromatography and tandem mass spectrometry. Mol Cell Proteomics 6: 1088-1102, 2007

24. Inci MF, Özkan F, Ark B, Vurdem ÜE, Ege MR, Sincer I and Zorlu A: Sonographic evaluation for predicting the presence and severity of coronary artery disease. Ultrasound Q 29: 125-130, 2013.

25. Crouse JR III, Grobbee DE, O'Leary DH, Bots ML, Evans GW, Palmer MK, Riley WA and Raichlen JS; METEOR Study Group: Carotid intima-media thickness in low-risk individuals with asymptomatic atherosclerosis: Baseline data from the METEOR study. Curr Med Res Opin 23: 641-648, 2007.

26. Cheng M, Li BY, Li XL, Wang Q, Zhang JH, Jing XJ and Gao HQ: Correlation between serum lactadherin and pulse wave velocity and cardiovascular risk factors in elderly patients with type 2 diabetes mellitus. Diabetes Res Clin Pract 95: 125-131, 2012.

27. Fu Z, Wang M, Gucek M, Zhang J, Wu J, Jiang L, Monticone RE, Khazan B, Telljohann R, Mattison J, et al: Milk fat globule protein epidermal growth factor-8: A pivotal relay element within the angiotensin II and monocyte chemoattractant protein-1 signaling cascade mediating vascular smooth muscle cells invasion. Circ Res 104: 1337-1346, 2009.

28. Gao BB, Stuart L and Feener EP: Label-free quantitative analysis of one-dimensional PAGE LC/MS/MS proteome: Application on angiotensin II-stimulated smooth muscle cells secretome. Mol Cell Proteomics 7: 2399-2409, 2008.

29. Hanayama R, Tanaka M, Miwa K, Shinohara A, Iwamatsu A and Nagata S: Identification of a factor that links apoptotic cells to phagocytes. Nature 417: 182-187, 2002.

30. Cunha C, Gomes C, Vaz A and Brites D: Exploring new inflammatory biomarkers and pathways during LPS-induced M1 polarization. Mediators Inflamm 2016: 6986175, 2016.

31. Redondo S, Navarro-Dorado J, Ramajo M, Medina Ú, Molina-Sanchez P, Garces Z, García-Alonso M, Reguillo F, Rodriguez E, Andres V and Tejerina T: Age-dependent defective TGF-betal signaling in patients undergoing coronary artery bypass grafting. J Cardiothorac Surg 9: 24, 2014.

32. Kanzaki T, Tamura K, Takahashi K, Saito Y, Akikusa B, Oohashi H, Kasayuki N, Ueda M and Morisaki N: In vivo effect of TGF-beta 1. Enhanced intimal thickening by administration of TGF-beta 1 in rabbit arteries injured with a balloon catheter. Arterioscler Thromb Vasc Biol 15: 1951-1957, 1995.

33. Nabel EG, Shum L, Pompili VJ, Yang ZY, San H, Shu HB, Liptay S, Gold L, Gordon D, Derynck R, et al: Direct transfer of transforming growth factor beta 1 gene into arteries stimulates fibrocellular hyperplasia. Proc Natl Acad Sci USA 90: 10759-10763, 1993.

34. Chen CC, Chow MP, Huang WC, Lin YC and Chang YJ: Flavonoids inhibit tumor necrosis factor-alpha-induced up-regulation of intercellular adhesion molecule-1 (ICAM-1) in respiratory epithelial cells through activator protein-1 and nuclear factor-kappaB: Structure-activity relationships. Mol Pharmacol 66: 683-693, 2004.

35. Ammirati E, Moroni F, Norata GD, Magnoni M and Camici PG: Markers of inflammation associated with plaque progression and instability in patients with carotid atherosclerosis. Mediators Inflamm 2015: 718329, 2015.

36. Steyers CM III and Miller FJ Jr: Endothelial dysfunction in chronic inflammatory diseases. Int J Mol Sci 15: 11324-11349, 2014. 